\title{
Avaliação de Desempenho de Funções de Base Radial Compacta na Formulação do Método dos Elementos de Contorno com Integração Direta
}

\author{
Abraão Lemos Caldas Frossard ${ }^{1}$ \\ Programa de Pós Graduação em Engenharia Mecânica, PPGEM/UFES, Vitória, ES \\ Carlos Friedrich Loeffler ${ }^{2}$ \\ Programa de Pós Graduação em Engenharia Mecânica, PPGEM/UFES, Vitória, ES
}

Resumo. Este trabalho tem como objetivo testar funções de base radial na técnica de solução do método dos elementos de contorno, denominada MECID, desenvolvida para aproximar os termos integrais não autoadjuntos, presentes em muitas equações diferenciais parciais. Esses termos podem representar ações de domínio como fontes, etc.. Aqui aborda-se a Equação de Helmoltz e o termo referente à inércia é aproximado por uma sequência de funções radiais. O MECID já foi empregado com êxito anteriormente usando funções mais simples. Espera-se que o uso de funções mais modernas e consistentes possa melhorar os resultados já obtidos.

Palavras-chave. Método dos Elementos de Contorno, Equação de Helmoltz, Funções Radiais

\section{Introdução}

Considere a Equação de Helmholtz em sua forma integral inversa [1], dada por:

$$
c(\xi) u(\xi)+\int_{\Gamma} u(X) q^{*}(\xi ; X) d \Gamma-\int_{\Gamma} q(X) u^{*}(\xi ; X) d \Gamma=\frac{1}{c^{2}} \omega_{n}^{2} \int_{\Omega} u(X) u^{*}(\xi ; X) d \Omega
$$

Onde $\mathrm{u}(\mathrm{X}), \mathrm{u}^{*}(\xi ; \mathrm{X}), \mathrm{q}(\mathrm{X})$ e $\mathrm{q}^{*}(\xi ; \mathrm{X})$ são: o potencial escalar, a solução fundamental e as respectivas derivadas normais; $\omega_{n}$ são as frequências naturais. O coeficiente $c(\xi)$ depende do posicionamento do ponto $\xi$ no domínio $\Omega(\mathrm{X})$. Na MECID, o núcleo completo da integral de domínio é interpolado através de funções radiais $F^{i}$, em que para cada ponto fonte $\xi$, a interpolação corresponde a uma varredura de todos os pontos base $X^{i} \mathrm{em}$ relação aos pontos $\mathrm{X}$ do domínio, ponderada pelos coeficientes ${ }^{\xi} \alpha^{j}$. Assim:

\footnotetext{
${ }^{1}$ abraaocaldas@hotmail.com

${ }^{2}$ carlosloeffler@bol.com.br
} 


$$
\int_{\Omega} u(X) u^{*}(\xi ; X) d \Omega=\int_{\Omega} \xi \alpha^{i} F^{i}\left(X^{i} ; X\right) d \Omega=\int_{\Omega} \xi \alpha^{i} \psi_{\prime j j}^{i}\left(X^{i} ; X\right) d \Omega={ }^{\xi} \alpha^{i} \int_{\Gamma} \eta^{i}\left(X^{i} ; X\right) d \Gamma
$$

Para transformar a integral de domínio em uma integral de contorno é adotada uma função de interpolação primitiva $\psi^{i}$ de $F^{i}$, seguido pela utilização do teorema da divergência. $\eta^{i}$ é o produto da derivada primeira de $\psi^{i}$ e do vetor normal ao contorno. Como as funções de interpolação radiais são arbitrárias, os coeficientes ${ }^{\xi} \alpha^{i}$ são incógnitos e podem ser obtidos através da solução do seguinte sistema de equações, em que figura uma matriz diagonal $\Lambda$, composta dos valores da solução fundamental:

$$
\left[{ }^{\xi} \alpha\right]=[F]^{-1}\left[{ }^{\xi} \Lambda\right][F] \alpha=[F]^{-1}\left[{ }^{\xi} \Lambda\right][u]
$$

\section{Funções de Interpolação}

Modernamente, com o desenvolvimento de técnicas de aproximação envolvendo multivariáveis, novas classes de funções de base radial foram utilizadas com êxito em problemas de interpolação, ajuste de curvas e solução de equações diferenciais parciais, particularmente nas modernas formulações sem malha. Interessa testar, sobretudo, as funções de base radial compacta com suporte pleno, pois tais funções possuem propriedades importantes: são positivas definidas e formam matrizes com diagonais dominantes, resultando maior precisão na solução do sistema de equações discretas. A Tabela 1 mostra algumas das funções de Wendland [3] de mais baixa ordem.

Tabela 1: Funções Radiais de Wendland.

\begin{tabular}{|c|c|}
\hline Função de Wendland de Suporte Compacto & \\
\hline$\Phi_{3,0}\left(\frac{r}{\delta}\right)=\left[1-\frac{r}{\delta}\right]_{+}^{2}$ & $C^{0} \cap P D_{3}$ \\
\hline$\Phi_{3,1}\left(\frac{r}{\delta}\right)=\tau^{1} \Phi_{3}\left(\frac{r}{\delta}\right)=\left[1-\frac{r}{\delta}\right]_{+}^{4}\left[1+4 \frac{r}{\delta}\right]$ & $C^{2} \cap P D_{3}$ \\
\hline$\Phi_{3,2}\left(\frac{r}{\delta}\right)=\tau^{2} \Phi_{4}\left(\frac{r}{\delta}\right)=\left[1-\frac{r}{\delta}\right]_{+}^{6}\left[35\left(\frac{r}{\delta}\right)^{2}+18 \frac{r}{\delta}+3\right]$ & $C^{4} \cap P D_{3}$ \\
\hline
\end{tabular}

\section{Referências}

[1] C.A. Brebbia, The Boundary Element Method for Engineers, Pentech Press, (1978).

[2] M.D. Buhmann, Radial Basis Function: Theory and Implementations, Cambridge Press, (2003).

[3] H. Wendland, Piecewise polynomial, positive definite and compactly supported radial functions of minimal degree, Adv. in Comput. Math., 4: 389-396, (1995). 